\title{
Telemental Health and the Management of Psychosis
}

\author{
Amy L. Donahue ${ }^{1} \cdot$ Jennifer Rodriguez ${ }^{1} \cdot$ Jay H. Shore ${ }^{1,2}$ \\ Accepted: 11 February 2021 / Published online: 24 March 2021 \\ (C) The Author(s), under exclusive licence to Springer Science+Business Media, LLC, part of Springer Nature 2021
}

\begin{abstract}
Purpose of Review The purpose of this paper is to review the application of telehealth in the assessment and treatment of psychotic illnesses. We present the contextual factors which make this approach to clinical care compelling, and review existing evidence about feasibility, acceptability, and effectiveness.

Recent Findings The use of telehealth with individuals that suffer from serious mental illness and psychosis has been demonstrated to be feasible and acceptable, with effectiveness that is comparable to in-person clinical care. Telehealth holds the additional promises of expanding access, connecting patients, families, and the general public to behavioral health resources, and reducing overall health care costs.

Summary We provide two case examples which demonstrate the successful use of technology for the delivery and coordination of effective patient care for individuals with psychotic illnesses.
\end{abstract}

Keywords Telemental health $\cdot$ Telepsychiatry $\cdot$ Psychotic illness $\cdot$ Feasibility $\cdot$ Acceptability $\cdot$ Effectiveness

\section{Introduction}

It is estimated that 1 in 5 adults in the USA, nearly 45 million people, lives with mental illness. Psychotic disorders alone have a lifetime prevalence of $3 \%$ [1]. Illness burden of such magnitude leads to profound human suffering and significant financial burden. The World Health Organization estimates that neuropsychiatric disorders lead to $33 \%$ of the global burden of total years lived with disability and an economic cost equivalent to approximately $2.5 \%$ of the

This article is part of the Topical Collection on Psychiatry in the Digital Age

Amy L. Donahue

amy.donahue@coaccess.com

Jennifer Rodriguez

jennifer.rodriguez@accesscare.com

Jay H. Shore

jay.shore@ cuanschutz.edu

1 Access Care Services, Colorado Access, 11100 E Bethany Drive, Aurora, CO 80014, USA

2 Department of Psychiatry and Family Medicine, School of Medicine And Centers for American Indian and Alaska Native Health, Colorado School of Public Health, Anschutz Medical Campus, Aurora, CO, USA
US gross national product [2-4]. Individuals with severe mental disorders, including schizophrenia and bipolar disorder, have also been shown to die 10 to 20 years earlier than the general population, and those with psychosis have the highest relative risk for increased mortality $[5,6]$. Longer duration of untreated psychosis is associated with cognitive deficits, reduced brain connectivity, more severe illness course, worse response to treatment, and decreased quality of life [7-12]. These poor outcomes make a patient linkage with specialty care providers essential to alter health outcome trajectories.

Unfortunately, it is estimated that nearly half of all adults in need of behavioral health care do not receive the needed treatment. There are numerous barriers to treatment for those suffering from mental illness. For those individuals with psychosis, when early symptoms may be subtle and difficult to detect, the nature of the illness can lead to a lack of insight and awareness about the need for treatment. In addition, mental health workforce shortages throughout the country have been well documented. According to a 2016 SAMSA report on rural behavioral health, it is estimated that more than $75 \%$ of all US counties are mental health workforce shortage areas and half of all US counties have no mental health professionals at all. Rurality and per capita income have been shown to be predictors of this shortfall $[13,14]$. The use of telehealth has emerged as one solution to improving access to behavioral health care [15]. 


\section{Telehealth as a Solution}

According to the American Telemedicine Association, telehealth can be defined as "technology-enabled health and care management and delivery systems that extend capacity and access" [16]. Services can be provided using an array of different formats and delivery systems, which allow for provider consultation, patient assessment and monitoring, ongoing treatment, and psychoeducation (Fig. 1).

Technology has been used in community and home-based care for centuries [17]. The US Department of Health and Human Services estimates that nearly half of all health care institutions and hospitals currently use some form of telehealth, and according to the American Medical Association, physician utilization of digital health has increased in recent years, particularly when it is viewed as a tool to improve efficiency and patient safety $[18,19]$. Reimbursement and parity laws also significantly influence the use the telehealth, which in the era of COVID-19 have improved under legislative changes and the emergency declaration status [20, 21]. In 2018, The American Psychiatric Association and the American Telemedicine Association released an updated practice guideline to inform best practice standards for videoconferencing-based telemental health $[22 \bullet \bullet$. The document provides important guidance for the development and implementation of a telemental health program, with recommendations drawn from the evidence base which supports the safety and efficacy of this approach to clinical care. Recent reviews have demonstrated the feasibility, acceptability, and effectiveness of telepsychiatry; however, many do not specifically focus on the potential benefits for individuals with psychosis [23, 24]. While psychotic illness tends to be less common than mental health conditions like depression and anxiety, the associated elevated risk of morbidity and mortality makes the application of telehealth in this population a worthwhile focus of study.

\section{Telehealth and Psychotic Illness}

There has been an international effort to systematically review the evidence base for the application of telehealth for individuals with severe mental illness and psychosis. While differing study methods and definitions of telehealth modalities at times make interpretation of the evidence challenging, there are clear efforts underway to broaden our understanding of the potential benefits of telehealth for the assessment and treatment of patients with psychosis $[25,26,27 \bullet \bullet, 28]$. One particularly useful review was published in 2016 by the European Psychiatric Association, which offered a set of 5 graded recommendations for telehealth interventions, based on the quality of the evidence to date [29•].

What appears to be consistently demonstrated in systematic reviews is the feasibility of delivering care via telehealth in its various forms, including the utilization of videoconferencing technology, internet-based programs, and mobile health applications [30, 31]. A finding of additional promise is the general acceptability of telehealth by both service users and providers. As it can be the nature of psychosis for individuals to have ideas of reference, it is an understandable concern that the use of videoconferencing could trigger an exacerbation of symptoms. Fortunately, with rare exception, this experience has not

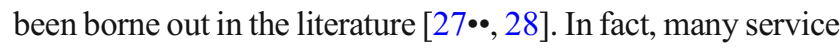
users have expressed preferring this type of delivery system due to the convenience. One could also speculate that the physical separation that is provided by telehealth can lead to a sense of security and control that could decrease anxiety for psychotic patients, as demonstrated in some other reports in telehealth treatments of anxiety [32].

Patient assessments that are conducted using videoconferencing have been found to be equivalent to face-to-face assessments in controlled studies, which speaks to the reliability of this approach. Reliability appears to be additionally enhanced when providers use a higher speed internet connection. The poorer quality of lower bandwidth has been shown to potentially impair the ability to assess the more subtle negative symptoms of schizophrenia, although this association has not been consistently demonstrated [26, 28].

Finally, the use of telehealth has been shown to lead to improvements in patient outcomes. The use of mobile health technology and telephonic interventions have been shown to lead to improved medication compliance and increased
Fig. 1 Examples of telehealth service

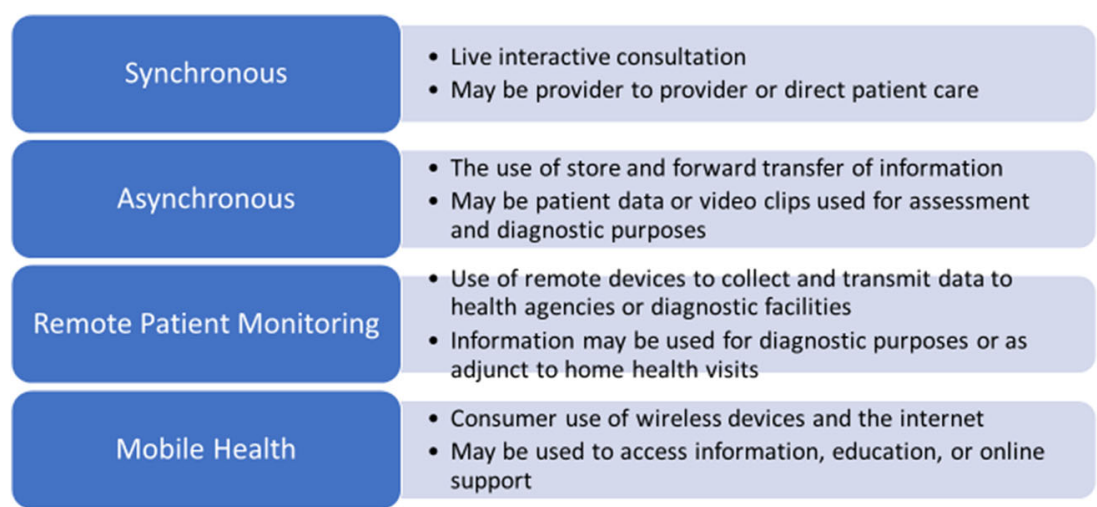


attendance at medication management appointments [25, 30]. A controlled study has demonstrated the reliability of administering the Abnormal Involuntary Movement Scale (AIMS) over videoconferencing, although in-person exams may be required to monitor other movement disorder symptoms not addressed in the AIMS (e.g., cogwheeling). Mobile health applications additionally show promise for symptom tracking, relapse prevention, and illness self-management [30-34]. Innovative programs have also been developed and have been shown to improve cognitive and social functioning for individuals with serious mental illness, including the application of virtual reality technology for social skills training and enhancement of work performance [25]. Patients have also reported a reduction in symptom severity when receiving care through telehealth, as measured by standardized assessment tools including the Brief Psychiatric Rating Scale (BPRS) and the Positive and Negative Syndrome Scale (PANSS). Improvements were found to be comparable to reported improvements when a patient received in-person care [28, 29•].

Below, we present two clinical cases from our work intended to illustrate common themes and issues that can arise when working with patients dealing with psychotic illness.

\section{Case Example 1}

"Jacob" is a 23-year-old male who was referred for psychiatric consultation following a hospital admission for treatment of an acute psychotic episode. At the time of his initial outpatient assessment, Jacob was residing in a small remote rural community.

Jacob's mother brought him to the appointment, and she provided background information. Jacob met with the psychiatrist via videoconferencing, and while he presented with grandiose delusional beliefs, he denied hallucinations and was able to provide basic information about his medication regimen. He was started on an oral antipsychotic medication in the hospital and due to his ongoing symptoms, the dose was increased and converted to a long-acting injectable formulation.

Jacob's psychiatrist and family worked with his primary care provider to coordinate needed laboratory monitoring and medication injections. Ancillary staff at the primary care provider's office were also able to call Jacob and his mother to remind them of appointments and facilitated linkage to a local mental health clinician to assist Jacob in meeting his goals of completing a GED and finding employment.

Throughout the course of his treatment, Jacob was consistent with follow-up appointments, was compliant with nursing visits and medication administration, presented clinically with a reduction in psychotic symptoms, and was able to sustain housing and regular employment.

This case helps to demonstrate the ability of telehealth to provide critical access to psychiatric services for patients dealing with psychosis in remote and rural communities. It also shows the criticality, and need of this case, to involve both family members and team members at the patient side to support compliance and case management functions to address both logistical treatment concerns (eg. labs and injections) and social determinants of health (eg. education and employment).

\section{Case Example 2}

"Paul" is a 17-year-old male who was referred for psychiatric consultation following a visit with his primary care provider, where he presented with auditory hallucination after reportedly smoking LSD-laced marijuana. His appointment was scheduled through his primary care provider, who had a relationship with a behavioral health clinician to provide diagnostic assessments and treatment via videoconferencing.

Paul was brought to the appointment by his paternal aunt, uncle, and older brother, with whom he was residing. They provided transportation and background information for the completion of the assessment. Paul's aunt reported that within the past week they had taken him to the emergency room because of his symptoms. They were told he should be better in a week or two and sent the family home. Paul then called the police twice during the past week due to auditory hallucinations, became increasingly paranoid, and locked himself in his room and at one point, jumped out the second-floor apartment window, and ran down the street. When his family caught up with him, he became physically aggressive toward his uncle. Paul's family reported concern for his safety and due to his increasing psychosis, they did not think they could continue to safely care for him.

Paul had difficulty engaging in the assessment with the telehealth clinician via videoconferencing. He appeared guarded and reported paranoid delusions that the telehealth clinician was recording the session. Paul also reported visual and command auditory hallucinations, seeing "shadows" and hearing the television telling him "don't go, run."

Paul's telehealth clinician, family, and primary care team worked together to transfer Paul to the hospital for additional assessment. Paul was admitted to an inpatient behavioral health unit where he was diagnosed with schizophrenia. $\mathrm{He}$ was started on effective treatment and his family was educated about his diagnosis, which enabled safe discharge to home with coordinated aftercare.

This second case differs from the first in the ability of the patient to cooperate and engage in the assessment as well as having the videoconference interplay with his psychotic symptoms. Despite this, telehealth played an important role in the necessary assessment, triage, and connection to care that led to a positive outcome during this episode. The telehealth behavioral health clinician had to assess the extent of Paul to engage in his assessment vs. working with the wider support 
of his family and team on the ground to manage the situation. The fundamental principles of engaging wider collaterals and on-site team members would not defer for the clinician had they been physically present with Paul. This counters the concerns of videoconferencing exacerbating or not being appropriate for use in psychosis especially if it gets incorporated into the psychotic processes.

\section{Discussion}

We proffer the following key considerations for clinicians and services working through telehealth with psychotic illnesses.

1. Create detailed clinical workflows, procedure, and protocols that account for the needs of this patient population. This includes having good safety protocols, knowing local commitment processes, connection to local resources and emergency services, and procedures for obtaining working with collateral contacts, and ordering and obtaining results of necessary testing (e.g., labs).

2. Assess and develop awareness of the patient's current mental status with attention to psychotic symptoms and whether these are involving the technology being used. Assess the patient's level of reality testing if they are experiencing psychotic symptoms involving technology, whether these are general or specific to the telehealth being used, and the patient's ability to distinguish these. If the patient is demonstrating difficulty with reality testing around technology, be sure to immediately engage local staff support and family members where clinically appropriate.

3. Leverage technology for gathering of collateral information and in-between patient session contacts. For the patient with chronic severe mental illness (SMI), consider using virtual home visits to assess function and care needs.

While technology has been used for centuries in the delivery of health care, it has not been until more recently that its application in the treatment of psychotic illnesses has been explored. Due to the increased risk for morbidity and mortality in individuals with SMI, it is worthwhile to explore potential opportunities to engage people with the needed resources and care. Associated increased rates of homeless and lower SES in individuals with SMI compound existing barriers to accessing mental health care and the current logistical challenges presented by COVID restrictions further threaten to widen the health disparity gap [35-37]. Findings suggest that the development and implementation of telehealth for the treatment of psychotic illnesses is feasible and acceptable and leads to outcomes which are aligned with best practices and standards of care for psychotic illnesses [38]. The use of telehealth and mobile devices has shown promise to meet the unique needs of patients with SMI, including mobile health programs that have delivered care to homeless youth, monitored suicidal ideation in patients with schizophrenia post-hospital discharge, and provided clozapine monitoring in the era of distance clinical care [39-41].

Not only can telehealth be used to improve access to behavioral health providers and quality care but has the potential to provide patients, families, and the general public with information that can be used to promote mental health awareness and early detection, assist with system navigation, and decrease the stigma surrounding mental illness [42•]. The use of telehealth also has the potential to decrease healthcare costs. Studies of health care utilization found decreased emergency room visits and inpatient days when psychotic patients received remote telemonitoring of medication compliance and symptoms when compared to care as usual [25]. Utilizing telehealth to integrate behavioral health care into the primary care setting, as reflected in the case examples, is an additional novel approach worth considering. The cost-saving benefits of integrated care have been well established through extensive actuarial analysis of the health care marketplace [43]. Integrated care provides benefits of improving linkage of patients with SMI, in lieu of the well-known findings that patients with SMI have shorter life spans due to excess mortality due to physical illness and are less likely to receive the same standard and quality of care [44].

It is important to balance the myriad potential benefits of telehealth with possible barriers to implementation. Practice transformation can be challenging, and successful implementation of telehealth programs requires buy in from key stakeholders including providers, health care systems, and users. Engagement can be facilitated by creating user-friendly systems and providing education about the value of technology and system integration for improving health outcomes.

\section{Conclusions}

The use of telehealth to provide care to individuals with psychotic illnesses is an important and worthwhile focus of study. While opportunities remain to build our evidence base, particularly separating out the application and efficacy of the various telehealth modalities for differing facets of care, there appear to be potential benefits for this approach to clinical care.

Acknowledgements The editors would like to thank Dr. Peter Yellowlees for taking the time to review this manuscript.

\section{Compliance with Ethical Standards}

Human and Animal Rights and Informed Consent This article does not contain any studies with human or animal subjects performed by any of the authors. 
Conflict of Interest Amy L. Donahue, Jennifer Rodriguez, Jay H. Shore work with AccessCare which provides Telemental Service in Colorado and Alaska.

\section{References}

Papers of particular interest, published recently, have been highlighted as:

- Of importance

•- Of major importance

1. Sullivan SA, Kounali D, Cannon M, David AS, Fletcher PC, Holmans $\mathrm{P}$, et al. A population-based cohort study examining the incidence and impact of psychotic experiences from childhood to adulthood, and prediction of psychotic disorder. Am J Psychiatry. 2020;177:308-17.

2. National Institute of Mental: Health Mental Health Information; 2019. [Internet] Available from: https://www.nimh.nih.gov/health/ statistics/mental-illness.shtml.

3. Health Resources \& Services Administration: Behavioral Health Workforce Projections; 2020. [Internet] Available from: https:// bhw.hrsa.gov/health-workforce-analysis/research/projections/ behavioral-health-workforce-projections.

4. Investing in Mental Health, 2003 [Internet]. World Health Organization. Available from: https://www.who.int/mental health/media/investing mnh.pdf.

5. Liu NH, Daumit GL, Dua T, Aquila R, Charlson F, Cuijpers P, et al. Excess mortality in persons with severe mental disorders: a multilevel intervention framework and priorities for clinical practice, policy and research agendas. World Psychiatry. 2017;16:30-40.

6. Walker ER, McGee RE, Druss BG. Mortality in mental disorders and global disease burden implications: a systematic review and meta-analysis. JAMA Psychiatry. 2015;72(4):334-41.

7. Oliver D, Davies C, Crossland G, Lim S, Gifford G, McGuire P, et al. Can we reduce the duration of untreated psychosis? A systematic review and meta-analysis of controlled interventional studies. Schizophr Bull. 2018;44(6):1362-72.

8. Penttila M, Jaaskelainen E, Hirvonen N, Isohanni, Miettunen J. Duration of untreated psychosis as predictor of long-term outcome in schizophrenia: systematic review and meta-analysis. Br J Psychiatry. 2014;205:88-94.

9. Maximo JO, Nelson EA, Armstrong WP, Kraguljac NV, Lahti AC. Duration of untreated psychosis correlates with brain connectivity and morphology in medication-Naïve patients with first-episode psychosis. Biol Psychiatry Cogn Neurosci Neuroimaging. 2020;5:2318 .

10. Sullivan SA, Carroll R, Peters TJ, Amos T, Jones PB, Marshall M, et al. Duration of untreated psychosis and clinical outcomes of first episode psychosis: an observational and an instrumental variables analysis. Early Interv Psychiatry. 2019;13:841-7.

11. Cotter J, Zabel E, French P, Yung AR. Early intervention in the real world. Prolonged duration of untreated psychosis: a problem that needs addressing. Early Interv Psychiatry. 2017;11:263-8.

12. Wang MY, Ho NF, Sum MY, Collinson SL, Sim K. Impact of duration of untreated psychosis and premorbid intelligence on cognitive functioning in patients with first-episode schizophrenia. Schizophr Res. 2016;175:97-102.

13. Rural Behavioral Health: Telehealth Challenges and Opportunities, 2016 [Internet]. Substance Use and Mental Health Services Administration. Available at: https://store.samhsa.gov/sites/ default/files/d7/priv/sma16-4989.pdf.
14. Behavioral Health in Rural America: Challenges and Opportunities, 2019 [Internet]. Rural Policy Research Institute. Available at: http:// www.rupri.org/wp-content/uploads/Behavioral-Health-in-RuralAmerica-Challenges-and-Opportunities.pdf.

15. Gardner JS, Plaven BE, Yellowlees P, Shore JH. Remote telepsychiatry workforce: a solution to psychiatry's workforce issues. Curr Psychiatry Rep. 2020;22(8).

16. American Telemedicine Association: Telehealth Basics; 2020 [Internet]. Available at: https://www.americantelemed.org/.

17. The Role of Telehealth in an Evolving Health Care Environment: Workshop Summary. Institute of Medicine of the National Academies; 2012.

18. Tuckson RV, Edmunds M, Hodgkins M. Telehealth. N Engl J Med. 2017;377(16):1585-92.

19. AMA Digital Health Research: Physicians' motivations and requirements for adopting digital health. Adoption and attitudinal shifts from 2016 to 2019. American Medical Association, February 2020

20. Harvey JB, Valenta S, Simpson K, Lyles M, McElligott J. Utilization of outpatient telehealth services in parity and nonparity states 2010-2015. Telemed J E Health. 2019;25(2):132-6.

21. Portnoy J, Waller M, Elliott T. Telemedicine in the era of COVID19. J Allergy Clin Immunol Pract. 2020;8:1489-91.

22.• Shore JH, Yellowlees P, Caudill R, Johnston B, Turvey C, et al. Best practices in videoconferencing-based telemental health April 2018. Telemed J E Health. 2018;24(11):1-6 Best practice guidelines for telemental health.

23. Bashshur RL, Shannon GW, Bashshur N, Yellowlees PM. Original research: The empirical evidence for telemedicine interventions in mental disorder. Telemed J E Health. 2016;22(2):87-113.

24. Hubley S, Lynch SB, Schneck C, Thomas M, Shore J. Review of key telepsychiatry outcomes. World J Psychiatry. 2016;6(2):26982.

25. Lawes-Wickwar S, McBain H, Mulligan K. Application and effectiveness of telehealth to support severe mental illness management: systematic review. JMIR Ment Health. 2018;5(4):e62.

26. Kasckow J, Felmet K, Appelt C, Thompson R, Rotondi A, Haas G. Telepsychiatry in the assessment and treatment of schizophrenia. Clin Schizophr Relat Psychoses. 2014;8(1):21-7.

27.• •• Santesteban-Echarri O, Piskulic D, Nyman RK, Addington JM. Telehealth interventions for schizophrenia-spectrum disorders and clinical high-risk for psychosis individuals: a scoping review. J Telemed Telecare. 2018;0(0):1-7 Updated review of the use of telehealth for individuals with psychotic illness.

28. Sharp IR, Kobak KA, Osman DA. The use of videoconferencing with patients with psychosis: a review of the literature. Ann General Psychiatry. 2011;10:14.

29. Gaebel W, Grossimlinghaus I, Kerst A, Cohen Y, HinscheBockenholt A, et al. European Psychiatric Association (EPA) guidance on the quality of eMental health interventions in the treatment of psychotic disorders. Eur Arch Psychiatry Clin Neurosci. 2016;266:125-37 Evidence-based recommendations for telehealth interventions.

30. Gire N, Farooq S, Naeem F, Duxbury J, McKeown M, et al. mHealth based interventions for the assessment and treatment of psychotic disorders: a systematic review. mHealth. 2017;3:33.

31. Amarendran V, George A, Gersappe V, Krishnaswamy S, Warren C. The reliability of telepsychiatry for a neuropsychiatric assessment. Telemed e-Health. 2011;17(3):223-5.

32. Yellowlees P, Shore JH (2018) Telepsychiatry and health technologies: a guide for mental health professionals. American Psychiatric Pub

33. Biagianti B, Schlosser D, Nahum M, Woolley J, Vinogradov S. Creating Live Interactions to Mitigate Barriers (CLIMB): a mobile intervention to improve social functioning in people with chronic psychotic disorders. JMIR Ment Health. 2016;3(4):e52. 
34. Bell HI, Lim MH, Rossell SL, Thomas N. Ecological momentary assessment and intervention in the treatment of psychotic disorders: a systematic review. Psychiatr Serv. 2017;68:1172-81.

35. Folsom DP, Hawthorne W, Lindamer L, Gilmer T, Bailey A, Golshan S, et al. Prevalence and risk factors for homelessness and utilization of mental health services among 10,340 patients with serious mental illness in a large public mental health system. Am J Psychiatry. 2005;162:370-6.

36. Werner S, Malaspina D, Rabinowitz J. Socioecenomic status at birth is associated with risk of schizophrenia: population-based multilevel study. Schizophr Bull. 2007;33(6):1373-8.

37. Zhai Y. A call for addressing barriers to telemedicine: health disparities during the COVID-19 pandemic. Psychother Psychosom 2020.

38. The American Psychiatric Association Practice Guideline For The Treatment of Patients with Schizophrenia, Third Edition. Copyright @ 20201 American Psychiatric Association.

39. Schueller SM, Glover AC, Rufa AK, Dowdle CL, Gross GD, et al. A mobile phone-based intervention to improve mental health among Homeless young adults: pilot feasibility trial. JMIR Mhealth Uhealth. 2019;7(7):e12347.

40. Kasckow J, Gao S, Hanusa B, Rotondi A, Chinman M, Zickmund $\mathrm{S}$, et al. Telehealth monitoring of patients with schizophrenia and suicidal ideation. Suicide Life Threat Behav. 2015;45(5):600-11.
41. Grover S, Mishra E, Chakrabarti S, Mehra A, Sahoo S. Telephonic monitoring of patients on clozapine in the resource-poor setting during the COVID-19 pandemic. Schizophr Res. 2020;222:48990.

42. Treisman GJ, Jayaram G, Margolis RL, Pearlson GD, Schmidt CW, et al. Perspectives on the use of eHealth in the management of patients with schizophrenia. J Nerv Ment Dis. 2016;204:620-9 Interesting perspective paper on the potential uses of telehealth from an expert panel of diverse stakeholders.

43. Melek SP, Norris DT, Paulus J, Matthews K, Waever A et al. Potential economic impact of integrated medical-behavioral healthcare. Milliman Research Report January 2018. Available at : file://C:/Users/AXDonahue/Downloads/Milliman-ReportEconomic-Impact-Integrated-Implications-Psychiatry.pdf.

44. De Hert M, Correll CU, Bobes J, Cetkovich-Bakmas M, Cohen DAN, Asai I, et al. Physical illness in patients with severe mental disorders. I. Prevalence, impact of medications and disparities in health care. World J Psychiatry. 2011;10(1):52.

Publisher's Note Springer Nature remains neutral with regard to jurisdictional claims in published maps and institutional affiliations. 